\title{
Status Epilepticus- An Atypical Presentation of Chorioamnionitis: A Case Report
}

\author{
Dr. Sadia Solaiman", Dr. Anupama Bondili \\ Women Health Institute, Al Ain Hospital, UAE
}

DOI: $10.36348 /$ sijog.2020.v03i07.004

| Received: 22.06.2020 | Accepted: 30.06.2020 | Published: 28.07.2020

*Corresponding author: Dr. Sadia Solaiman

\section{Abstract}

The objective of this clinical case report is to highlight an uncommon presentation of chorioamnionitis as status epilepticus, complicated by consumptive coagulopathy and recurrent hypoglycemia. A primigravida of 18 weeks gestation, presented to the Accident and emergency department via ambulance with seizures. She did not have any handheld medical records. No history could be obtained as she is a visitor with no friends and family. She was disoriented with Glasgow coma scale of 10/15, random blood sugar of 1.8 , blood pressure $88 / 58$, pulse 130 , and temperature $36.8^{\circ} \mathrm{C}$. Scan showed an 18 weeks live fetus. She was having uncontrollable seizures even after her glucose was corrected. Patient was also started on magnesium sulphate and anti epileptic infusions. She was intubated and transferred to ICU. On vaginal examination smelly discharge was noticed. Patient was started on Broad spectrum antibiotics. Her WCC elevated to 23 , other investigations like Liver function tests, urea \& electrolytes and creatinine were normal. The next day patient aborted spontaneously and continued to be tachycardic, tachypneic and hyperthermic with labs showing a picture of consumptive coagulopathy. Urine, blood culture and CSF cultures were normal. After Inotropes, antiepileptic's, platelet transfusion, broad spectrum antibiotics, and appropriate fluid management, patient's condition improved. Placental histopathology confirmed chorioamnionitis. She required ventilator support for 13 days. She suffered with recurrent episodes of hypoglycemia even after her sepsis was resolved which was treated by dextrose infusion and steroids and resolved spontaneously. She was discharged on $44^{\text {th }}$ day of her hospital stay in stable condition.

Keywords: Status epilepticus, Chorioamnionitis, Septic shock, Disseminated intravascular coagulation, hypoglycemia.

Copyright @ 2020: This is an open-access article distributed under the terms of the Creative Commons Attribution license which permits unrestricted use, distribution, and reproduction in any medium for non-commercial use (NonCommercial, or CC-BY-NC) provided the original author and source are credited.

\section{INTRODUCTION}

Chorioamnionitis presenting as status epilepticus has not been cited in any literature so far! Clinical chorioamnionitis is the most common infection related diagnosis made in labor and delivery units worldwide. It is traditionally believed to be due to microbial invasion of the amniotic cavity, which elicits a maternal inflammatory response characterized by maternal fever, uterine tenderness, maternal tachycardia and leukocytosis [1].

Chorioamnionitis frequently occurs in parallel with microbial infection of the chorioamnion and amniotic fluid .However, it may also occur in the absence of demonstrable microorganisms [2]. As in our case where all the cultures were negative.
Affected mothers have an increased rate of endometritis, post-operative wound infections, septic pelvic thrombophlebitis, postpartum hemorrhage, pelvic abscess, and other complications [3].

However, septic shock, disseminated intravascular coagulation, adult respiratory distress syndrome and maternal death are only rarely encountered [4].

Status epilepticus itself being rare occurs in only $1-2 \%$ of pregnancies [5], Chorioamnionitis with sepsis could be one of the differential diagnosis for status epilepticus in a pregnant woman as we present in this case. 


\section{CASE DESCRIPTION}

A 26-year-old primigravida, Ugandan female, domestic worker by profession and 18 weeks pregnant presented with history of 2 episodes of new onset seizures witnessed by co-workers and the paramedics respectively. Upon arrival her Glasgow coma scale was $10 / 15$ with a random blood sugar of 1.8 , blood pressure $88 / 58$, pulse 130 , temperature $36.8^{\circ} \mathrm{C}$ and urine protein $3+$. Scan showed an 18 weeks live fetus. Upon clinical examination: No neck rigidity, no signs of meningeal irritation, reflexes were normal, no clonus. All other systems were reviewed and was found negative. She received dextrose infusion to correct her hypoglycemia even after which she was having continuous uncontrolled seizures. Was started on mgso4 and phenytoin infusion. After receiving diazepam and Keppra, her seizures controlled. She was intubated and transferred to ICU for ventilatory support.

Once stabilized, vaginal examination revealed cervix dilated to $1 \mathrm{~cm}$ with foul smelling discharge. Labs showed aspartate aminotransferase and alanine aminotransferase at 122 units/L and 64 units/L respectively, white cell count of $20.3 \times 10^{\wedge} 9$, C-reactive protein of 156.3 and procalcitonin of $20 . \mathrm{Mgso} 4$ was started suspecting atypical eclampsia but within a few hours MGso4 was discontinued as the patient was having hypotensive episodes and picture was less likely of pre-eclampsia.

Electroencephalogram revealed no epileptiform activity, magnetic resonance imaging and magnetic resonance venography showed no abnormalities, Computed tomography of the brain was clear. She was started on Vancomycin +ceftriaxone+acyclovir+metronidazole.

The next day patient aborted spontaneously and continued to be tachycardic, tachypneic and hyperthermic with labs showing platelets of $42 \times 10^{\wedge} 9$, INR 1.5 and fibrinogen of 5.20, with a picture of consumptive coagulopathy. After Inotropes, platelet transfusion, broad spectrum antibiotics, and appropriate fluid management, patient's condition improved. Disseminated intravascular coagulation managed. She required ventilator support for 13 days.

Smear for plasmodium and acid-fast bacilli were negative, no toxins were detected in toxicology screen. High vaginal swab and Cerebrospinal fluid cultures showed no organisms. All other extrauterine causes of infection were ruled out. The above antibiotics were discontinued after the Cerebrospinal fluid culture came negative and was started on meropenem in view of suspected listerosis.

Placental histopathology confirmed chorioamnionitis, but placental smear for listeria came negative as well. She suffered with recurrent episodes of hypoglycemia even after her sepsis was resolved which was treated by dextrose infusion and steroids. Random cortisol level, Glycosylated hemoglobin level, insulin like growth factor 2 , thyroid function tests, Cpeptide level, CT abdomen, insulin antibodies and endoscopic ultrasound, all were done to find the cause for hypoglycemia but were inconclusive.

She was discharged on $44^{\text {th }}$ day of her hospital stay in stable condition and was lost to follow up.

\section{DISCUSSION}

Chorioamnionitis presenting as a status epilepticus is first ever case reported in the literature. Pregnancy-associated SE predominantly occurred in patients without a history of epilepsy [6]. The first task was differentiating this case from eclampsia, which is a more common cause of seizures in pregnancy. She had no history of hypertension or hypertensive disorders in pregnancy. Also, examination findings with absence of pedal edema as well as a normal blood pressure throughout her admission also hinted against eclampsia, thus despite a proteinuria of $3+$ the diagnosis of status epilepticus was favored. The additional knowledge that status epilepticus can cause proteinuria would also aid the understanding of the spectrum of her illness [7].

Seizures caused by hypoglycemia is less likely in this case, as even after correction of hypoglycemia siezures persisted ,and later post abortion while she had recurrent hypoglycemic episodes she remained seizure free.

In addition to eclampsia, other conditions such as viral encephalitis, systemic lupus erythematosus, cavernoma, reversible cerebral vasoconstriction syndrome, pyridoxine deficiency, and N-methyl-Daspartate (NMDA) receptor antibody mediated autoimmune encephalitis have been implicated to cause Status epilepticus in pregnancy [8]. In addition to above the other differentials considered were drug overdose, malaria, tuberculosis and septic abortion. In our case all investigations into these causes came as normal.

Even after an extensive work up, cause for Hypoglycemic episodes were not known. Insulinoma was considered one of the main differentials for the recurrent hypoglycemia, as many pregnant patients with insulinoma are misdiagnosed and treated with anticonvulsant therapy. Because of the often confusing clinical picture of pregnant women with insulinoma, a possibility of it has to be taken into account in the differential diagnosis of personality and consciousness changes during the first trimester of pregnancy or in the postpartum period [9] other Differentials for the hypoglycemia included hypocortisolemia, insulinoma, IGF-2 mediated hypoglycemia. 


\section{CONCLUSION}

Physicians should be vigilant about atypical etiologies of seizures during pregnancy. Chorioamnionitis with sepsis could be one of the differential diagnosis for status epilepticus in a pregnant woman. The risk of life-threatening maternal sequelae, such as sepsis, coagulopathy, and adult respiratory distress syndrome related to chorioamnionitis are low if treatment with broad-spectrum antibiotics is initiated [10].

As in our case, early recognition, careful and effective management with multidisciplinary involvement is necessary for good outcome.

This case reports the unexpected association between chorioamnionitis and status epilepticus. Possible follow-up with case series and subsequently higher level works (beyond case reports) would help in answering these emerging questions.

\section{REFERENCES}

1. Romero, R., Gomez-Lopez, N., Kusanovic, J.P., Pacora, P., Panaitescu, B., Erez, O., \& Yoon, B. H. (2018 June). Clinical chorioamnionitis at term: New insights into the etiology, microbiology, and the fetal, maternal and amniotic cavity inflammatory responses. Retrieved from https://www.ncbi.nlm.nih.gov/pmc/articles/PMCC6 177213/

2. Sweeney, E. L., Dando, S. J., Kallapur, S. G., \& Knox, C. L. (2017). The human Ureaplasma species as causative agents of chorioamnionitis. Clinical microbiology reviews, 30(1), 349-379.

3. Oh, K. J., Kim, S. M., Hong, J. S., Maymon, E., Erez, O., Panaitescu, B., ... \& Yoon, B. H. (2017). Twenty-four percent of patients with clinical chorioamnionitis in preterm gestations have no evidence of either culture-proven intraamniotic infection or intraamniotic inflammation. American journal of obstetrics and gynecology, 216(6), 604e1.

4. Tita, A. T., \& Andrews, W. W. (2010). Diagnosis and management of clinical chorioamnionitis. Clinics in perinatology, 37(2), 339-354.

5. Beach, R. L., \& Kaplan, P. W. (2008). Chapter 15 Seizures in Pregnancy. Diagnosis and Management. In B. Gidal, \& C. Harden (Eds.), Epilepsy in Women The Scientific Basis for Clinical Management (pp. 259-271). (International Review of Neurobiology; Vol. 83).

6. Lu, Y. T., Hsu, C. W., Tsai, W. C., Cheng, M. Y., Shih, F. Y., Fu, T. Y., Chuang, Y. C., \& Tsai, M. H. (2016). Status epilepticus associated with pregnancy: A cohort study. Epilepsy \& behavior : E\&B, 59, 92-97.

7. Obikili, C. G., Kahansim, M. L., \& Ocheke, A. N. (2019). Clinical improvement of status epilepticus after delivery: A case report. Tropical Journal of Obstetrics and Gynaecology, 36(2), 304-307.

8. Rajiv, K. R., Menon, R. N., Sukumaran, S., Cherian, A., Thomas, S. V., Nair, M., \& Radhakrishnan, A. (2018). Status epilepticus related to pregnancy: Devising a protocol for use in the intensive care unit. Neurology India,66(6), 1629.

9. Tomazic, M., Janez, A., \& Oblak, M. R. (2017). Hypoglycemia identified by a continuous glucose monitoring system in a second-trimester pregnant woman with insulinoma: a case report. Journal of medical case reports, 11(1), 117.

10. Chen, K. T., Ramin, S. M., \& Barss, V. A. (2012). Intrapartum fever. UptoDate. 